\title{
Model identification of bridges using measurement data
}

\author{
Y. Robert-Nicoud, B. Raphael, O. Burdet and I.F.C. Smith \\ EPFL-Swiss Federal Institute of Technology \\ Structural Engineering Institute \\ CH-1015 Lausanne \\ Switzerland
}

\begin{abstract}
Measurements are increasingly used to augment traditional assessments of structural state. Measurements of deflections, rotations and strain provide indications of damages as well as changes in the values of parameters such as Young's modulus. Finite element model updating methods have been developed in the 1990s for identifying structural state from measurements. Currently most methods aim to determine the values of stiffness coefficients that result in measured responses. In the present work, a model calibration method identifies causes of structural behaviour such as support conditions and material properties. Static measurement data is employed for model calibration. A case study of the Lutrive bridge in Switzerland illustrates the methodology. Candidate models whose responses reasonably match measured data are identified. These models are then examined in order to determine whether the calibrated values are physically possible. Such examinations lead to either model rejection or further measurements.
\end{abstract}

\section{Introduction}

Traditionally structures are inspected by experts in order to determine whether they are capable of satisfying strength and serviceability criteria. An increasing number of structures are being equipped with measurement systems in order to augment traditional assessments and to increase understanding of structural behavior. Although measurements of deflections, rotations and strain provide indications of damages as well as gradual deterioration in the structural state, interpretation of measurements for obtaining metrics that directly indicate structural health or damage is not a wellestablished science (Housner et al., 1997).

When a structure is monitored periodically, changes in deflection patterns are potentially useful for determining whether material properties change with respect to time and also to examine whether there is any damage after critical events. This process is costly since measurements need to be taken several times a year. Another complication is that deflection patterns are influenced by factors such as temperature changes. However, if appropriate corrections to such effects are applied, data from continuous monitoring, along with appropriate modeling, help determine gradual and sudden changes in the structural state. 
When it is not possible to take measurements several times a year, modeling techniques remain useful for determining the structural state using a limited number of tests. Such modeling may employ system identification methods. These methods aim to compute the values of system parameters through a comparison of predicted and observed responses. When the form of the relationship between observable quantities and system parameters are known, regression techniques can be applied for identifying system parameters. The strategy is to minimize the error between the predicted output and measured values. This approach is common in dynamic systems that are employed in electrical and mechanical engineering. Standard techniques exist for selecting the model structure (form of the equation) from a set of candidate models (Ljung 1999). However, these techniques are not applicable to structural engineering systems because responses are highly non-linear with respect to model parameters.

Structures are usually analyzed by the finite element method for a known set of values of parameters such as material properties, geometric properties and loading in order to compute deflections and other responses such as mode shapes and frequencies. However, there is rarely a reverse procedure that directly computes the material and geometric properties from responses such as deflections. Procedures that aim to estimate these quantities through minimizing the error between measured and computed responses are known as finite element model updating techniques.

Currently, the most common method of damage detection using measurements is through the use of vibration data. This is an active research area. Friswell and Mottershead (1995) provide a survey of model updating procedures using vibration measurements. Recent papers published in this area include, Brownjohn et al. (2003), Castello et al. (2002), Teughels et al. (2002), Modak et al. (2002), Hemez and Doebling (2001), Sohn and Law (2001), Hu et al. (2001), Reich and Park (2001), Chaudhary et al. (2000), Yu et al. (1999), Abe (1998), Beck and Katafygiotis (1998), Katafygiotis and Beck (1998), Park and Felippa, (1998), and Sohn and Law (1997). Most work in this area aim to compute the set of stiffness coefficients that produce observed vibration modes of structures. Location and extent of damage are inferred through a comparison between the stiffness coefficients of the damaged and undamaged structures.

Since model-updating procedures are generally developed for specific types of responses, these procedures are not applicable to other response types. For example, problem formulations have been developed for computing the set of stiffness coefficients that produce observed vibration modes of structures. These formulations cannot be used to determine unknown forces when there is uncertainty with respect to the loading and environmental factors. Also, most methodologies are not able to determine causes of abnormal structural behavior, such as settlement of supports and cracking of concrete sections.

There are isolated reports of work involving static measurements to interpret structural state. Model updating procedures using static measurements either use deflection data or strain data. Papers that discuss model updating using deflection data include, Hajela and Soeiro (1990), Sanayei and Onipede (1991), Sanayei and Scamoli (1991), Banan and Hjelmstad (1994 a,b), Hjelmstad and Shin (1997). Sanayei and Saletnik (1996) and Liu and Chian (1997) are examples of work involving model updating using strain measurements. All of this work involves 
formulation of appropriate optimization problems for the minimization of the error between analytical and measured quantities for a given finite element model. The number of unknown variables is fixed in each optimization problem. Models that have varying number of degrees of freedom and consequently, different sets of variables are not accommodated in such approaches.

In structural engineering, models cannot always be approximated by closed form mathematical expressions and therefore, it is not possible to apply mathematical methods for minimization of errors, such as those described in textbooks, for example (Ljung 1999). Mathematical methods are not applicable when classes of models containing different degrees of freedom (and consequently different forms of equations) need to be searched to locate the minimum. In such situations, it is necessary to utilize optimization methods that employ a "generate-test" methodology (Raphael and Smith, 2003a). Optimization methods that make use of only the values of the objective function and do not require additional information such as gradients are generally known as direct search methods (Lewis et al. 2000). Points are generated and tested for the satisfaction of objectives and constraints through the evaluation of the objective function. The most widely used direct search techniques are simulated annealing (Kirkpatrick et al. 1983, Martin, 1995), and genetic algorithms (Holland 1975, Goldberg, 1989). Koh et al., (2003) use a combination of genetic algorithms and local search techniques for the identification of structural parameters using vibration measurements. Sharman and Esparcia-Alcazar (1993) use a combination of genetic algorithm and simulated annealing to optimize both the model structure and the numerical parameters of the model. Search techniques have also been combined with qualitative reasoning for system identification. Recent examples are found in Bradley et al. (2001) and Kay et al. (2000). Dunn (1998) uses a combination of genetic algorithm and stochastic hill climbing to compute the stiffness coefficients of a mass-spring system. Cunha et al. (1999) use genetic algorithm to estimate the stiffness coefficients by minimizing a residual formed from the Eigen solutions. Another example of the use of genetic algorithm for the determination of stiffness reduction is Mares and Surace (1996). The main drawback of the above methodologies is that causes of structural behavior cannot be easily determined using the values of stiffness coefficients.

This paper describes an approach to making use of measurement data for performing diagnostic assessments of structures. The overall project goal is to identify possible causes of observed behaviour. Since it is not often possible to make definite conclusions related to causes, the focus of the current study involves identifying candidate models of behaviour. Model predictions that match measured values as closely as possible are identified. An examination of the characteristics of these models leads to explanations of observed behaviour. The outline of this paper is as follows: measurement systems that have been used in the present study are discussed in Section 2; the approach based on model identification and calibration is discussed in Section 3; results are summarized in Section 4.

\section{Measurement systems}

Measurements that are useful for making diagnostic assessments of structures are examined in this section. Three types of measurement systems have been used in the present study. They are: 
- Hydrostatic leveling

- Inclinometers

- Fiber optic sensors

Appropriate interpretations of the data that is collected by these systems are necessary for structural evaluations. When applicable, such interpretations are discussed with the descriptions given below.

\subsection{Hydrostatic leveling}

The hydrostatic leveling system (Burdet 1993, Favre et al. 1995) is based on the principle of communicating vessels. The deflection at any point in the structure is obtained by computing the sums and differences of measured levels at various points. This system has the following advantages:

- It is easy to install and is inexpensive

- Discontinuous operations are possible, permitting the reactivation of an unused system after years of discontinued service, provided that new tubing is installed.

- Operation is possible even under heavy traffic, as the system exhibits a large inertia.

Among the main disadvantages of most installed systems are:

- Impossibility to obtain continuous measurements: human intervention is necessary for taking measurements

- The system cannot be used below $0^{\circ} \mathrm{C}$ since the liquid used is pure demineralized water.

- The accuracy of the measurement is proportional to the number of intermediate readings to reach a given point. Typical values for the accuracy are 0.5 to $3 \mathrm{~mm}$.

- A large number of circuits may be needed in bridges with longitudinal slopes.

- It is easily applicable only to box girder bridges having accessible boxes.

This system has been successfully installed in more than 10 bridges in Switzerland. In some cases, it is operated by the local bridge authority, and in other cases the measurements are made by a university research team (Favre et al. 1995). Under normal operating conditions, measurements are taken three times a year, in the spring, summer and fall.

\subsection{Inclinometers}

Inclinometers measure rotations within a structure. A property of measuring a structure's rotations is that, for a given ratio of maximum deflection to span length, the maximum rotation is independent of details of the static system (Daverio 1995). Inclinometers used in this study are Wyler Zerotronic $\pm 1^{\circ}$ (Wyler 1996). Their accuracy is 1 microradian ( $\mu \mathrm{rad}$ ), which corresponds to a rotation of one millimeter per kilometer. For an intermediate span of a continuous beam with a constant depth, a mid-span deflection of 1/20,000 would induce a maximum rotation of about $150 \mu \mathrm{rad}$, or 0.15 milliradians (mrad). Advantages of inclinometers are:

- The measure is relative only to the initial position. In case of failure of an instrument, the information given by the others is still usable. 
- Inclinometers are electronic instruments that produce electric signals easily captured by standard data acquisition systems. The inclinometers used include an industrial network interface (RS 485) which reduces the amount of cabling.

- The amplitude of rotations is essentially independent of the static system or the cross section of a bridge. Because of their reliability, inclinometers can be located in hard to reach places, and are thus suitable for all kinds of cross sections.

- The high accuracy of inclinometers makes it possible to measure very small movements, such as those that occur within a couple of minutes.

- Inclinometers are very compact instruments that require only a minimal space. Some models include automatic temperature compensation. The sensors can be easily replaced and are reusable.

- The cost of inclinometers is competitive with comparable systems, and the cost of installation is low.

Figure 1 shows a simple installation of an inclinometer on a fixed support.

\subsubsection{Reconstruction of the deflected shape}

Although rotations can give valid indications on the condition of a structure, it is generally necessary to reconstruct the deflected shape of the structure from the measured rotations to permit an easier interpretation. Once rotation values have been acquired, their values are combined to reconstruct the corresponding deflected shape. The simplest approach consists of fitting a polynomial through the measured values. Three inclinometers define a second order polynomial. Higher order polynomials can be fitted with more inclinometers. By integrating the polynomial, and applying the boundary conditions, the deflected shape is obtained. Even though reconstruction of the deflected shape by simple polynomials has been found to be effective, its practical application is difficult due to sensitivity to measurement errors since the polynomial is forced to pass through all the measured values. Least squares fit might be used to reduce the sensitivity to measurement errors. However results are unlikely to be accurate since theoretical deflections for structures with continuously varying cross section properties cannot be described by simple functions.

Considering these weaknesses another approach was used. Deflected shapes are reconstructed by means of linear combinations of a series of pre-calculated deflected shapes (Burdet and Zanella, 2000). Structures deflect following smooth "engineer's curves" under standard load cases (Figure 2). A linear combination of a certain number of these curves, yields a curve that is also a smooth curve. Each deflected shape respects the bridge properties, and can account for discontinuities, such as sudden changes of inertia, much better than polynomials.

Figure 3 shows the deflected shape that is reconstructed using pre-calculated deflected shapes (base shapes). Also shown in the figure is the shape that is obtained by integrating a fourth degree polynomial and applying the boundary condition that there are no displacements at both supports. Both curves lie close to deflection data that are measured directly.

A feature of the method of using precalculated deflected shapes is that the reconstructed deflected shape yields additional information on the way the best 
deflected shape is obtained. For example, a case where all combinations of two of the four loading patterns of Figure 2 are used, the combination that gives the best result is $10 \%$ of uniform loading plus $102 \%$ of unit load at $0.75 \mathrm{~L}$, where $\mathrm{L}$ is the span. The interpretation of these coefficients, and their variation over time provides additional information about the behavior of the bridge. It is for example expected that significant changes in the structure will lead to significantly different factors used in the controlling combination, thus indicating that the structure has changed.

Results obtained by applying this procedure to inclinometer measurements taken during a load test on Lutrive bridge (See Section 3.1) are shown in Figure 4. Computed deflections have a good match with those that are directly measured using optical leveling instruments.

\subsection{Fibre optic sensors (SOFO)}

The SOFO (Surveillance d'Ouvrage par Fibre Optique) system is based on low coherence interferometry in standard optical fibres that are used in telecommunication (Inaudi et al. 1994). The system measures small deformations and provides a resolution of approximately 2 microns. A significant advantage of the system compared with conventional strain gauges is that long gauge lengths are possible and therefore, local effects such as cracking do not influence measurements. Also, since the system uses light rather than electricity, long term absolute measurements are more reliable. The system has been successfully installed in many bridges.

Internal deformations are difficult to interpret. Therefore, the deflected shape of bridged are computed from internal deformations measured by SOFO sensors through the following procedure (Vurpillot et al., 1998):

- The bending curvature is computed from strain measurements assuming that plane sections remain plane after bending (Bernoulli beam hypothesis). If two fibre optical sensors are placed on the upper part and lower parts of a beam element, the curvature is computed using this relationship:

$\frac{1}{\mathrm{r}_{m}}=\frac{l_{\text {inf }}-l_{\text {sup }}}{Y \cdot l_{1}}$

where,

$r_{m}$ is the mean radius of curvature

$l_{1}$ is the initial length of upper and lower sensors

$l_{\text {sup }}$ is the final length of upper sensor

$l_{\text {inf }}$ is the final length of lower sensor

$Y$ is the distance between upper and lower sensors

- The curvature is integrated twice and appropriate boundary conditions are applied to obtain the deflected shape.

Figure 5 shows a comparison between the deflection shape retrieved using this procedure and direct measurements using strain gauges.

\section{Model identification and calibration}


Currently, measurements are mostly used for continuously monitoring deflections in order to detect changes in structural behavior. In this work, measurements are used to improve understanding of the behavior of the structure. A strategy that makes use of measurements to improve model identification and calibration is described in this section.

The main objective of this study is to identify characteristics of behavior models that result in measured responses. Unlike recent work on model updating, the goal is not to find values of stiffness coefficients that produce measured responses. Instead, we are interested in identifying reasons for structural behavior such as support settlements and cracks. Sets of models are searched and sets of parameter values are computed such that the residual errors between predictions and measurements are minimized.

The fundamental strategy is "generate and test" as described in Section 1. Most importantly, this strategy involves searching among diverse models that have varying number of degrees of freedom and do not have explicit closed form mathematical expressions for responses. A search algorithm called PGSL (Probabilistic Global Search, Lausanne) (Raphael and Smith, 2003b) was used for finding sets of combinations of values of model parameters that produce close matches to measurements. PGSL performs global search by sampling the solution space using a probability density function (PDF). At the beginning of search, a uniform PDF is assumed for the entire search space so that solutions are generated purely randomly. When good solutions are found, probabilities in those regions are increased so that more intense sampling is carried out in the neighborhood of good solutions. The key assumption is that better sets of points are found in the neighborhood of good sets of points. The search space is gradually narrowed down so that convergence is achieved.

The PGSL algorithm includes four nested cycles (Figure 6a):

- Sampling cycle

- Probability updating cycle

- Focusing cycle

- Subdomain cycle

In the sampling cycle (innermost cycle) a certain number of samples, NS, are generated randomly according to the current PDF. Each point is evaluated by the user-defined objective function and the best point is selected. In the next cycle, probabilities of regions containing good solutions are increased and probabilities decreased in regions containing less attractive solutions (Figure 6b). In the third cycle, search is focused on the interval containing the best solution after a number of probability updating cycles, by further subdivision of the interval (Figure 6c). In the subdomain cycle, the search space is progressively narrowed by selecting a subdomain of smaller size centred on the best point after each focusing cycle. This is done by multiplying the current width of each axis by a scale factor. 
During model calibration, PGSL minimizes the root mean square error (RMSE), which is calculated using the following equation:

$R M S E=\sqrt{\frac{\sum_{i}\left(x_{i, c}-x_{i, m}\right)^{2}}{n}}$

where $x_{i, m}$ is the value measured at the $\mathrm{i}$-th measurement point and $x_{i, c}$ is the corresponding value computed using the model. The search routine identifies combinations of values of model parameters that produce low values of RMSE. Since PGSL is based on global sampling there is no need to provide initial values to search variables. Bounds of variables are specified based on engineer's expectations of possible variations in values.

As an alternative to the procedures described in Sections 2.2.1 and 2.3, models once calibrated may be employed to compute deflected shapes from measured slopes and internal deformations. First, candidate models are identified in which predicted slopes and strains match measurements. Next, these models are analyzed to obtain the deflected shape. Through this procedure, assumptions related to the degree of polynomial need not be made and the deflected shapes conform to the laws of structural mechanics.

The Lutrive bridge example is taken next to illustrate the model identification and calibration strategy. The measurement event that is evaluated is a load test that was conducted in 1997. The advantage of this case is that knowledge of the loading, including magnitude and position on the structure, is available.

\subsection{Lutrive Bridge}

The Lutrive highway bridge was constructed in 1972 using the cantilever method with central hinges (articulations). Two bridges, (North and South Lutrive bridges) were built (one for each direction of traffic) with a length of $395 \mathrm{~m}$. each and a maximum span of approximately $130 \mathrm{~m}$. The longitudinal section is shown in Figure 7. The cross-section of the bridge is a pre-stressed box-girder with variable inertia. The maximum height is $8.50 \mathrm{~m}$. at the column and $2.50 \mathrm{~m}$. in the mid-span, at the hinges. At the location of the hinge, the deck is reduced to $0.14 \mathrm{~m}$, without interrupting the continuity of the beam (Figure 7c). More information about the bridge can be found in [Burdet and Badoux, 1999]. A brief history of the bridge is given below:

1971-1972

1973, 77, 78, 80, 85, 86

1986

December 1988

1988-1999

November 1997
Construction of the bridge

Annual optical level meter measurements

An engineering office was given the contract to survey the bridge after large deformations were observed at midspan

External pre-stress is added to the two bridges

Displacement on the south bridge increases. New measurement systems are installed on both bridges, including a hydrostatic leveling system (1988), and fiber optical sensors (1996)

Load tests with fiber optic sensors, inclinometers, and 
December 1999

optical level meter

New additional external pre-stress added to the south bridge

\subsection{Load tests on Lutrive bridge}

Finding a good explanation for abnormal increases in deflections in the Lutrive bridge has remained a challenge for several years. Various hypotheses involving parameters such as creep, pre-stress and models of joints at mid-span have been made. In order to improve understanding of the behavior of the bridge and to find out causes for abnormal deflections, load tests were carried out on the bridge in 1997.

One section of the bridge was equipped with instruments (Figure 7a). During the load test, a truck was placed on the part of the bridge that was instrumented in order to produce maximum deflections at the locations of sensors. The weight of the truck was approximated to be a two-point load placed non-symmetrically (Figure 7b).

\subsubsection{Measurement system}

The following measurement systems were used during the load tests.

- Fiber optic sensors at the following five longitudinal positions (Perregeaux 1998) from the column in the Lausanne direction: 8.4m., 20.4 m., 32.5 m., 44.5 m., 56.8 m.

- Inclinometers at four positions from the same column (Burdet and Fleury, 1997):

16.1 m., 29.5 m., 49.3 m., 65.75 m.

- Optical level meters at four positions from the same column (Burdet and Fleury, 1997):

11.9 m., 29.9 m., 47.8 m., 65.75 m.

The following measurement data are obtained from the measurement systems:

- Deformations of sensors placed on the top and bottom of the bridge define the bending curvature.

- Inclinometers give the slope of the cross-section.

- Optical level meters give the vertical displacement of cross-sections.

\subsection{Model identification}

Initially, simple models were used to interpret the measurements from load tests. When simple models were found to be inadequate to explain the results, more complex models containing additional variables were created. Values of selected variables were determined through identifying low values of the root mean square error, see Equation 2. A sample set of models are discussed next. Only the part of the bridge from the column to the mid-span (the part that was equipped with measurement instruments) was modeled with models A, B, C, and D (Figure 8). The complete bridge excluding the columns was modeled using $\mathrm{E}$. 
Even though, PGSL is able to search automatically among classes of models that have varying numbers of degrees of freedom, in this work, model classes are manually selected and PGSL is used only for estimating values of parameters. The goal is to study the capability of each model class to explain measured data. Variables have been chosen such that there is a unique global minimum for each model class within specified variable bounds and the current study is not affected by the influence of local minima.

\subsubsection{A sample set of models}

\subsubsection{Model A: Cantilever}

This is a simple model that was used in a previous study (Perregeaux, 1998). The objective of using this model is to evaluate the boundary case of a cantilever bridge using expected values of material properties. It was assumed that there is no shear and moment transfer through the hinge from the other side. The structural system is a cantilever with parabolic section profile (variable moment of inertia). The value of Young's modulus (E) is taken to be $38 \mathrm{e} 6 \mathrm{kN} / \mathrm{m} 2$. Since there were no unknown variables, no calibration was used and the structure was evaluated analytically. This model did not achieve an acceptable correlation with measurements. There was more than $200 \%$ error in the mid-span deflection.

\subsubsection{Model B: Propped cantilever}

In order to investigate the effect of shear transfer from the other side of the hinge, a propped cantilever model was used in which a spring is used to support the beam at the propped end. The stiffness of the spring was estimated to be $17^{\prime} 114 \mathrm{kN} / \mathrm{m}$. No calibration was used since all the parameters were assigned constant values. This model produced a close match with measured deflections. However, predictions of curvatures did not match the measurements.

\subsubsection{Model C: Beam with springs}

Since the predictions of curvature using model B did not match measured values, it was suspected that the bending moments in the beam did not correspond to what was predicted using a propped cantilever model. Therefore, additional springs were added to adjust the boundary conditions of the model. Specifically, a horizontal spring was added at the midspan to model the arch effect caused by the variation in the position of the neutral axis. The spring stiffnesses were considered to be variables and possible values were identified using PGSL. Three separate searches were carried out to minimize the RMSE between predicted and observed deflections, slopes and curvatures respectively. The search using measured deflections produced a good match with respect to deflections. However, the calibrated model did not produce matching slope or curvature. Similarly, minimization of RMSE in slopes and curvatures did not produce a good match with respect to measurements that were not used in the calibration. The values of variables determined through this strategy are given in Table 1. 


\subsubsection{Model D: Beam supported on springs with rigid body rotation}

When Model C was calibrated using curvature measurements, slopes were found to differ from measured values by a constant value. Therefore, a variable representing rigid body rotation at the left support was added to the model. Variables of this model were then determined through minimizing the RMSE in slope. The values of these variables are given in Table 1 . There is a good match with deflections even though deflection measurements were not used in the calibration. Since possible explanations for the rigid body rotation (-0.072) could not be accepted this model was discarded.

\begin{tabular}{|c|l|c|c|c|c|c|}
\hline Model & Measurements & \multicolumn{5}{|c|}{ Values of variables in the model found by PGSL } \\
\cline { 3 - 7 } & $\begin{array}{l}\text { used for } \\
\text { calibration }\end{array}$ & $\begin{array}{c}\mathrm{E} \\
{\left[\mathrm{kN} / \mathrm{m}^{2}\right]}\end{array}$ & $\begin{array}{c}\mathrm{k} \\
{[\mathrm{kN} / \mathrm{m}]}\end{array}$ & $\begin{array}{c}\mathrm{k} 1 \\
{[\mathrm{kNm} / \mathrm{rad}]}\end{array}$ & $\begin{array}{c}\mathrm{k} 2 \\
{[\mathrm{kN} / \mathrm{m}]}\end{array}$ & $\begin{array}{c}\theta_{\text {rig }} \\
{[\mathrm{mrad}]}\end{array}$ \\
\hline \hline C & deflection & $50.00 \mathrm{e} 6$ & $28{ }^{\prime} 076$ & $9.99 \mathrm{e} 19$ & $2.8 \mathrm{e}-8$ & - \\
\hline C & slope & $28.34 \mathrm{e} 6$ & $15^{\prime} 625$ & $9.96 \mathrm{e} 19$ & $2.64 \mathrm{e}-4$ & - \\
\hline C & curvature & $48.64 \mathrm{e} 6$ & $16^{\prime} 300$ & $9.81 \mathrm{e} 19$ & $5.48 \mathrm{e} 5$ & - \\
\hline D & curvature & $48.64 \mathrm{e} 6$ & $16^{\prime} 300$ & $9.81 \mathrm{e} 19$ & $5.48 \mathrm{e} 5$ & -0.072 \\
\hline
\end{tabular}

Table 1: Model parameters determined by optimization

\subsubsection{Model E: Finite element model of the entire structure}

Examination of model D made it clear that boundary conditions are important. Therefore, the entire structure was modeled and analyzed using finite element method. Since models C and D highlighted the importance of varying the position of the neutral axis in order to produce an arch effect, (especially at the location of the hinge), this feature was included in the model. The hinge was modeled using a beam element with reduced value of the stiffness. The Young's modulus of concrete was calibrated in order to produce a good match with measurements. The final value obtained is $30 \mathrm{e} 6 \mathrm{kN} / \mathrm{m}^{2}$. This model produced good match with deflections and reasonable match with slope and curvature. Therefore, this is the most attractive candidate model found in this study.

\subsubsection{Summary of results}

Values of displacement, slope and curvature that are predicted by models and measured on the structure are shown in Figures 9-11. Each curve shows the prediction of a candidate model along the length of the beam and is obtained be interpolating values computed at measurement points. For finite element models, values are computed at gauss points nearest to measurement points.

\subsubsection{Analysis of results}

The results are used to classify models based on the percentage deviation (PD) which is computed by dividing the root mean square error by the mean value of data points. 
Models are classified as good (PD <25\%), reasonable (PD 25-50 \%) and bad (PD > $50 \%$ ) as shown in Table 2.

\begin{tabular}{|c|c|c|c|}
\hline \multirow{2}{*}{$\begin{array}{l}\text { Model } \\
\text { Measurement used for calibration } \\
\text { is given in brackets }\end{array}$} & \multicolumn{3}{|c|}{ Criteria } \\
\hline & Displacement & Slope & Curvature \\
\hline$\overline{\mathrm{A}}$ & Bad & 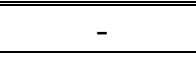 & 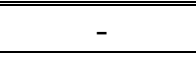 \\
\hline $\mathrm{B}$ & Good & Reasonable & Bad \\
\hline C (deflection) & Good & Bad & Reasonable \\
\hline C (slope) & Bad & Good & Reasonable \\
\hline C (curvature) & Bad & Reasonable & Good \\
\hline D (curvature) & Good & Good & Good \\
\hline $\mathrm{E}$ & Good & Reasonable & Reasonable \\
\hline
\end{tabular}

Table 2: Evaluation of models

The following observations are made from this study:

- Selecting models through reference to only one type of observation is not sufficient. It is easy to obtain models whose variable values provide a close match with one type of measurement through the model calibration strategy. However, these models may not be compatible with other types of measurements.

- When finite element models are used, a small change in boundary conditions greatly affects the results. When Model E was modified such that the hinge position coincides with the neutral axis, results were not satisfactory and therefore, they are not presented in this paper.

- The process of identifying models that are compatible with measurements may lead to examining models that have large numbers of variables. Model D contained 5 variables. The number of observation points must be greater than the number of parameters. A better match with more parameters does not necessarily mean that the quality of the model is better.

- Model calibration is useful for validating models as well as filtering out bad models. For example, Model C did not give good results for all three types of calibration attempts.

- Curvature data measurements show a change in sign near the hinge indicating that the mid-span joint transfers shear stress. This is not possible with Model A.

- Quantitative information related to values of parameters is more difficult to verify: For instance, Young Modulus of Model E is $30 \mathrm{e} 6 \mathrm{kN} / \mathrm{m}^{2}$ whereas it is 48.6e $6 \mathrm{kN} / \mathrm{m}^{2}$ for Model D.

- Models can only be accepted if sound physical explanation is found. Even though Model D resulted in a good match with all types of measurements, it was not accepted because a physical explanation could not be found for the calibrated value of rigid body rotation.

Predictions of Model E match well with all measurements. Model E is equivalent to Model D within the part of the bridge that is instrumented since for Model E, spring stiffnesses and rotations are influenced by other parts of the bridge. However, model $\mathrm{E}$ does not involve arbitrary stiffness coefficients and rigid body rotations as does 
model D. Correct modeling of the hinge is necessary to produce good predictions; the location of the hinge should be above the neutral axis of the beam and the hinge should be capable of transferring both horizontal and vertical forces. Mid span deflection may more than double if the hinge is incapable of transferring these forces. Therefore, it is hypothesized that hinge characteristics have changed over time in the Lutrive bridge and this has caused mid span deflections to increase.

\section{Summary and conclusions}

The following conclusions are drawn from this study:

- Measurements of deflections, rotations and internal deformations in concrete structures are now more reliable with the development of new equipment such as fibre optic sensors and inclinometers. Periodic monitoring allows detection of abnormalities. However, model identification techniques are needed for determining likely causes of abnormalities.

- It is possible to compute deflections from measured rotations and internal deformations. Thus, one type of measurement may be validated through the use of other types of independent measurements. Techniques that are available for computing the deflected shapes from rotations and internal deformations include integration of approximate polynomials, superposition of deflected curves and model calibration.

- Model calibration techniques lead to identification of good candidate models.

- Models that are identified through calibration have to be carefully studied by engineers in order to determine whether they are physically possible. All models should have sound physical support. When physical justifications cannot be found, models should be rejected. Alternately, further observations could reveal sufficient support for some candidate models.

- PGSL is an effective global search algorithm for model calibration tasks. In all the models of Lutrive bridge that were considered, PGSL was able to find parameter values that predicted a good match with the measurement data that was used in calibration.

- A plausible explanation for the increased deflection of the Lutrive bridge is a change in centre span hinge characteristics.

Further work is necessary to determine the mechanisms which have lead to changes in hinge characteristics. Possible explanations involved plasticity induced by prestress and subsequent stress cycles due to temperature variations.

This work has already been extended to search among multiple model classes using PGSL for system identification. A study of the reliability of system identification using this procedure is also in progress.

\section{References}

Abe M. (1998), Vibration control of structures with closely spaced frequencies by a single actuator, Journal of Vibration and Acoustics, Transactions of the American Society of Mechanical Engineers, Vol.120, pp.117-124. 
Banan M.R., Banan M.R., and Hjelmstad K.D. (1994a), Parameter estimation of structures from static response. Part 1. Computational aspects, Journal of structural engineering, ASCE, 120(11), pp. 3243-3258.

Banan M.R., Banan M.R., and Hjelmstad K.D. (1994b), Parameter estimation of structures from static response. Part 2. Numerical simulation studies, Journal of structural engineering, ASCE, 120(11), pp. 3243-3258.

Beck J.L. and Katafygiotis L.S. (1998), Updating Models and Their Uncertainties. I: Bayesian statistical framework, Journal of Engineering. Mechanics. 124, pp. 455-461.

Brownjohn J.M.W, Moyo P., Omenzetter P., Lu Y. (2003), Assessment of highway bridge upgrading by testing and finite-element model updating. Journal of bridge engineering, ASCE, 37, pp. 162-172.

Bradley E., Easley M., and Stolle R. (2001), "Reasoning about nonlinear system identification," Artificial Intelligence, 133:139-188.

BURDET O. (1993), Load Testing and Monitoring of Swiss Bridges, Comité Européen du Béton, Safety and Performance Concepts, Bulletin d'information n²19, Lausanne, Suisse.

Burdet O. and Badoux M.,(1999), Long-term Deflection Monitoring of Prestressed Concrete Bridges Retrofitted by External Post-Tensioning - Examples from Switzerland, IABSE Symposium "Structures for the Future - The Search for Quality", Rio de Janeiro, Report Vol 83, pp.112-114.

Burdet O., Fleury B. (1997), Pont sur la Lutrive aval (VD)- Rapport d'essai de charge statique complémentaire, EPFL-IBAP.

Burdet O., Zanella J.-L. (2000), Automatic Monitoring of Bridges using Electronic Inclinometers, IABSE, , Lucerne Congress Structural Engineering for Meeting Urban Transportation Challenges, pp. 398-399, Zürich, Switzerland, September.

Castello D.A., Stutz L.T., Rochinha F.A. (2002), A structural defect identification approach based on a continuum damage model, Computers and structures, 80, pp. 417-436.

Chaudhary M.T.A., Abe M, Fujino Y., and Yoshida J. (2000), Performance evaluation of two base-isolated bridges using seismic data, Journal of Structural Engineering, ASCE, Vol.116, No.10, pp.1187-1195.

Cunha J., Cogan S., Berthod C. (1999), Application of genetic algorithms for the identification of elastic constants of composite materials from dynamic tests, International Journal for Numerical Methods in Engineering, 45, 7, pp. 891-900.

Daverio R. (1995), Mesures des déformations des ponts par un système d'inclinométrie, Rapport de maîtrise EPFL-IBAP, Lausanne, Switzerland. 
Dunn S. A. (1998), The use of genetic algorithms and stochastic hill-climbing in dynamic finite element model identification, Computers \& Structures, 66, 4, pp. 489497

Favre R., Burdet O., Charif H., Hassan M., Markey I.F. (1995), Enseignements tirés d'essais de charge et d'observations à long terme pour l'évaluation des ponts en béton et le choix de la précontrainte, Office Fédéral des Routes, recherche 83/90, Suisse.

Fest E. (1997), Système de mesure par inclinométrie: développement d'un algorithme de calcul des flèches, DEA Ecole Normale supérieur de Cachan, France.

Friswell M.I. and Mottershead J.E. (1995), Finite element model updating in structural dynamics, Kluwer.

Goldberg, D.E. (1989), Genetic Algorithms in Search, Optimization, and Machine Learning, Addison-Wesley, Reading, MA.

Hajela P. and Soeiro F. J. (1990), Structural damage detection based on static and modal analysis," AIAA Journal, Vol. 28, No. 6, pp 1110 - 1115.

Hemez F.M., Doebling S.W. (2001), Review and assessment of model updating for non-linear, tansient dynamics, Mechanical Systems and Signal Processing, Vol 15(1), pp.45-74.

Holland J. (1975), Adaptation in natural artificial systems, University of Michigan Press.

Hjelmstad K.D., and Shin S. (1997), Damage detection and assessment of structures from static response, Journal of Engineering Mechanics, pp. 568-576.

Housner G. W., Bergman L. A., Caughey T. K., Chassiakos A. G., Claus R. O., Masri S. F., Skeleton R. E., Soong T. T., Spencer B. F., and Yao J. T. P. (1997), Structural control: past, present, and future, Journal of Engineering Mechanics, ASCE, Vol. 123(9), pp. 897-972.

Hu N., Wang X., Fukunaga H., Yao Z.H., Zhang H.X. and Wu Z.S. (2001), Damage assessment of structures using modal test data, International Journal of Solids and Structures, 38, pp. 3111-3126.

Inaudi D., Elamarib A., Pflug L., Gisinb N., Breguetb J., Vurpillot S. (1994), Lowcoherence deformation sensors for the monitoring of civil-engineering structures, Sensors and Actuators A 44, pp. 125-130.

Katafygiotis L.S. and. Beck J.L. (1998), Updating models and their uncertainties. II: model identifiability, Journal of Engineering Mechanics, 124, pp. 463-467.

Kay H., Rinner B., and Kuipers B. (2000). Semi-quantitative system identification. Artificial Intelligence, 119, pp. 103-140. 
Kirkpatrick, S., Gelatt C. and Vecchi M. (1983). Optimization by simulated annealing, Science, pp. 220:673.

Koh C.G., Chen Y.F., Liaw C.-Y. (2003), A hybrid computational strategy for identification of structural parameters, Computers and Structures, 81, pp. 107-117.

Liu P. and Chian C. (1997), Parametric identification of truss structures using static strains, ASCE Journal of Structural Engineering, July 1997, 123, 7, pp. 927-933.

Lewis, R.M., Torczon, V. and Trosset, M.W. (2000), Direct search methods: then and now, Journal of Computational and Applied Mathematics, 124, 191-207.

Ljung L. (1999), System Identification - Theory For the User, Prentice Hall.

Mares, C., Surace, C. (1996), Application of genetic algorithms to identify damage in elastic structures, Journal of Sound and Vibration, 195, 2, pp. 195-215.

Martin O. (1995), Combining simulated annealing with local search heuristics, Metaheuristics in combinatoric optimization, (G.Laporte and I.Osman editors).

Modak S.V., Kundra T.K., Nakra B.C. (2002), Comparative study of model updating studies using simulated experimental data, Computers and Structures, 80, pp. 437447.

Park K.C. and Felippa, C.A. (2001), A flexibility-based inverse algorithm for identification of structural joint properties, In Proceedings of ASME symposium on computational methods on inverse problems, 15-20 November 1998, Anaheim, CA.

Perregeaux N. (1998), Pont de la Lutrive-N9, Equipement et analyse du comportement au moyen du système de mesure à fibre optique SOFO, Diploma Thesis, EPF-Lausanne.

Perregaux N., Vurpillot S., Tosco J-S., Inaudi D., Burdet O. (1998), Vertical displacement of bridges using the sofo system: a fiber optic monitoring method for structures, ASCE-12th Engineering Mechanics. Conference Proceedings: A force for the 21st Century, 791-794, San Diego, USA,.

Raphael B. and Smith I.F.C. (2003a), Fundamentals of computer aided engineering, John Wiley, UK.

Raphael B. and Smith I.F.C. (2003b), "A direct stochastic algorithm for global search”, J of Applied Mathematics and Computation, Vol 146, No 2-3, pp 729-758.

Reich, G. W. and Park, K. C. (2001), A theory for strain-based structural system identification, Journal of Applied Mechanics, 68(4), 521-527.

Sohn H., and Law K.H. (1997), A Bayesian probabilistic approach for structural damage detection, Earthquake engineering and structural dynamics, 26, PP. 12591281. 
Sohn H., and Law K.H. (2001), "Damage diagnosis using experimental ritz vectors," Journal of Engineering Mechanics, ASCE, Vol. 127, No. 11, pp. 1184-1193.

Sanayei, M., and Onipede, O. (1991), "Damage assessment of structures using static test data," AIAA Journal, Vol.29, No.7, pp.1174-1179.

Sanayei, M., and Scampoli, S. (1991), "Structural element stiffness identification from static test data," Journal of Engineering Mechanics, ASCE, pp. 1021-1036.

Sanayei, M., and Saletnik, M. J. (1996), "Parameter estimation of structures from static strain measurements; I: formulation," Journal of Structural Engineering, ASCE, Vol. 122, No. 5, pp. 555-562.

Sanayei, M., and Saletnik, M. J. (1996), "Parameter estimation of structures from static strain measurements. II: error sensitivity analysis," Journal of Structural Engineering, ASCE, Vol. 122, No. 5, pp. 563-572.

Teughels A., Maeck J., Roeck G. (2002), Damage assessment by FE model updating using damage functions, Computers and structures, 80, pp. 1869-1879.

Yu L., Law S.S., Link M., Zhang L.M. (1999), damage detection in bolted joint structures using element contribution to modal strain energy, In Proceedings of the Second International Conference on Identification in Engineering Systems, Swansea, M.I. Friswell, J.E. Mottershead and A.W. Lees (eds.), pp. 516-526,.

Vurpillot S., Krueger G., Benouaich D., Clément D., Inaudi D. (1998), "Vertical deflection of a pre-stressed concrete bridge obtained using deformation sensors and inclinometer measurements", ACI Structural Journal, Vol 95, No 5, p 518 - 526.

Wyler AG. (1996), Technical specifications for Zerotronic Inclinometers, Winterthur, Switzerland. 


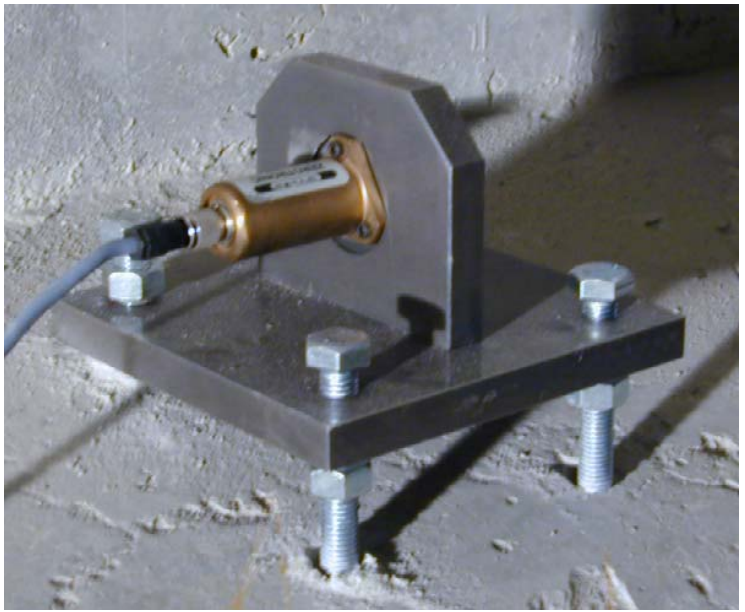

Figure 1 Simple support for a fixed inclinometer 


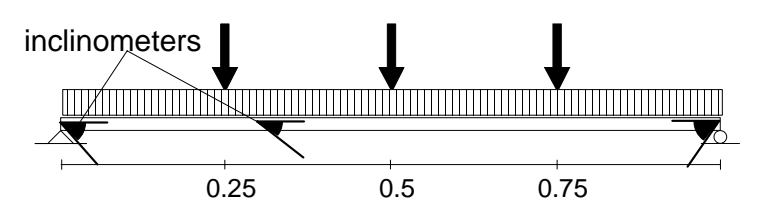

Figure 2 Load cases used for the pre-calculation of the base shapes 


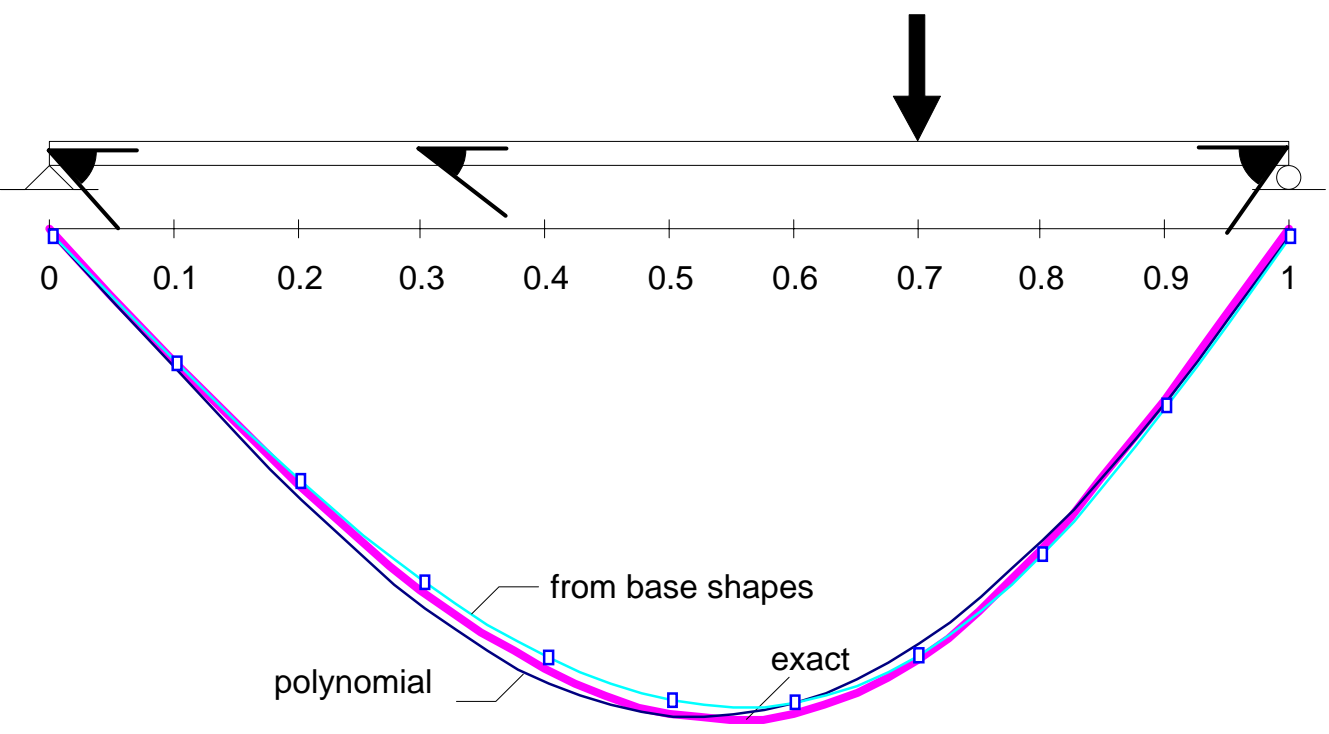

Figure 3 Deflected shapes by fitting a polynomial and by combinations of base shapes 


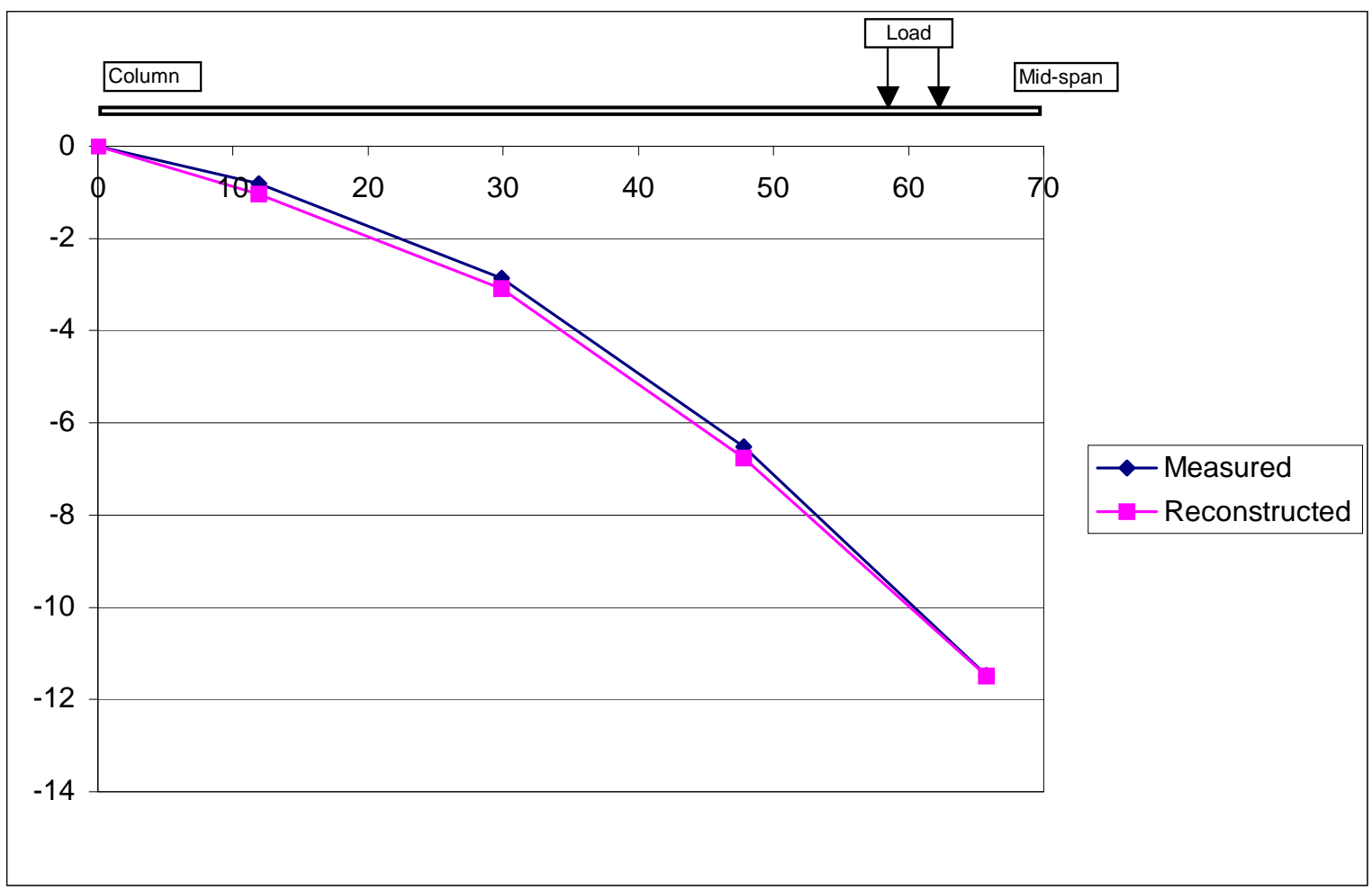

Figure 4 Deflections on Lutrive bridge calculated using superposition of deflected shapes 


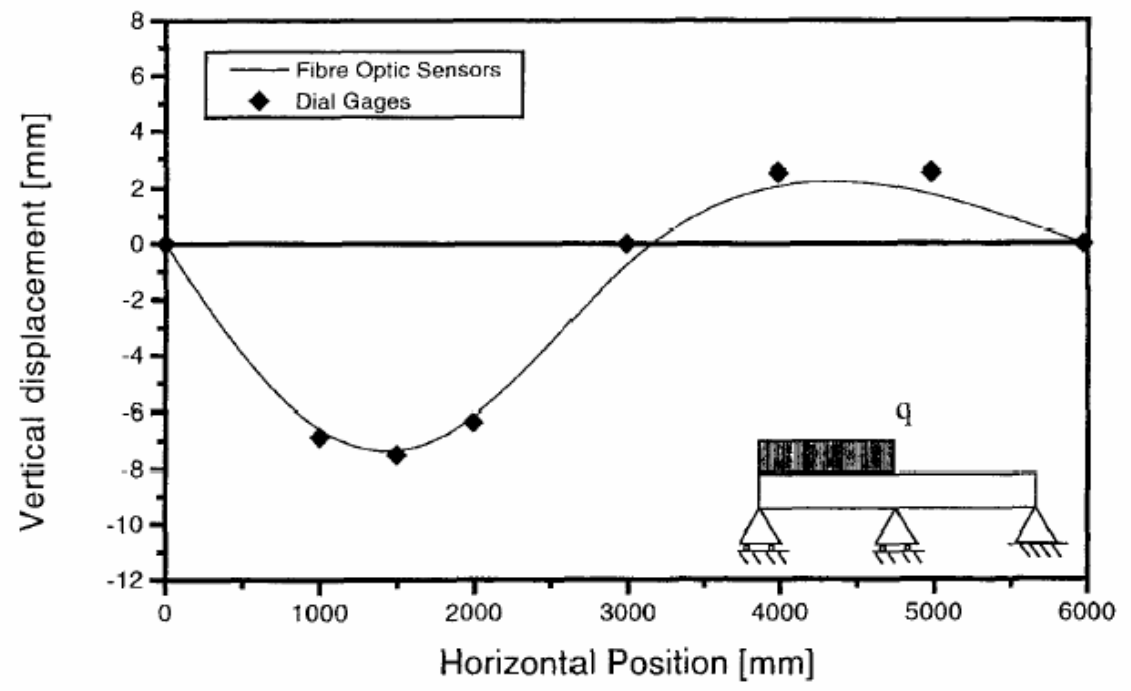

Figure 5 Comparison between vertical deflection retrieved from SOFO measurements and those measured by dial gauges (Vurpillot et al. 1998). 


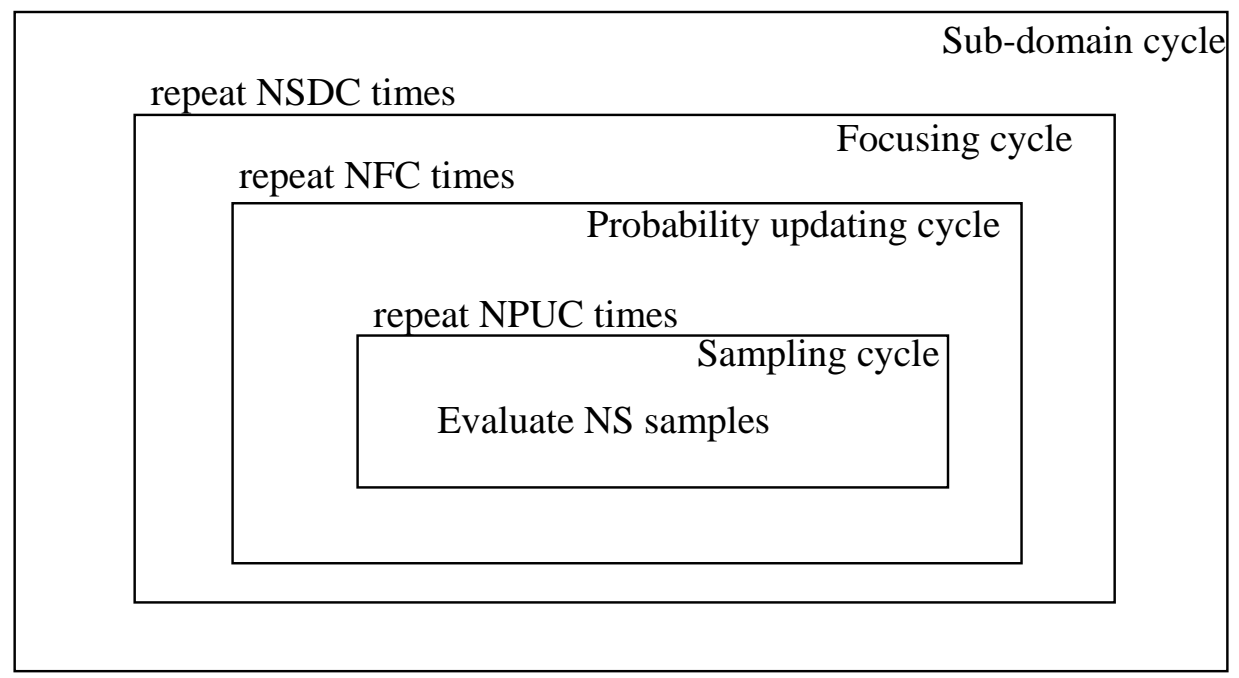

a) Nested cycles in PGSL. NSDC, NFC, NPUC and NS are the number of iterations in the subdomain, focusing, probability updating and sampling cycles respectively.

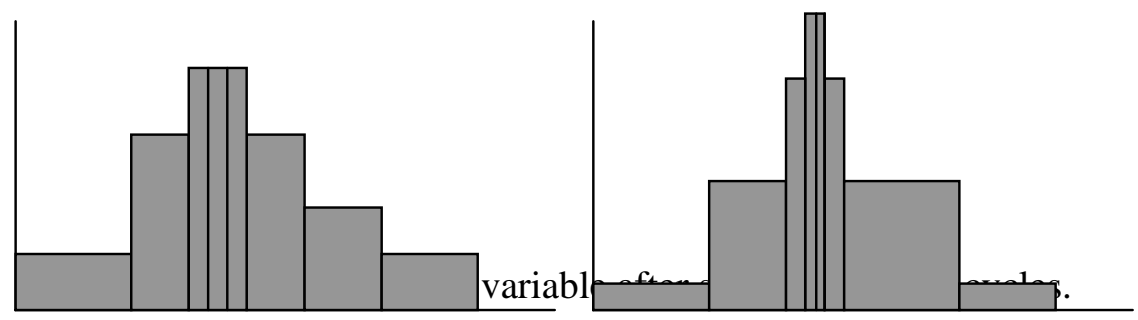

Best points in each cycle

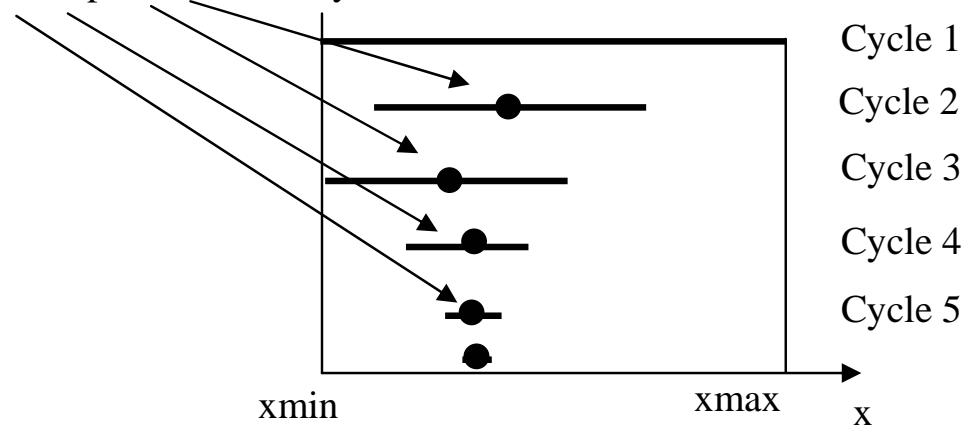

c) Changing the sub-domain after each subdomain cycle.

Figure $6 \quad P G S L$ 

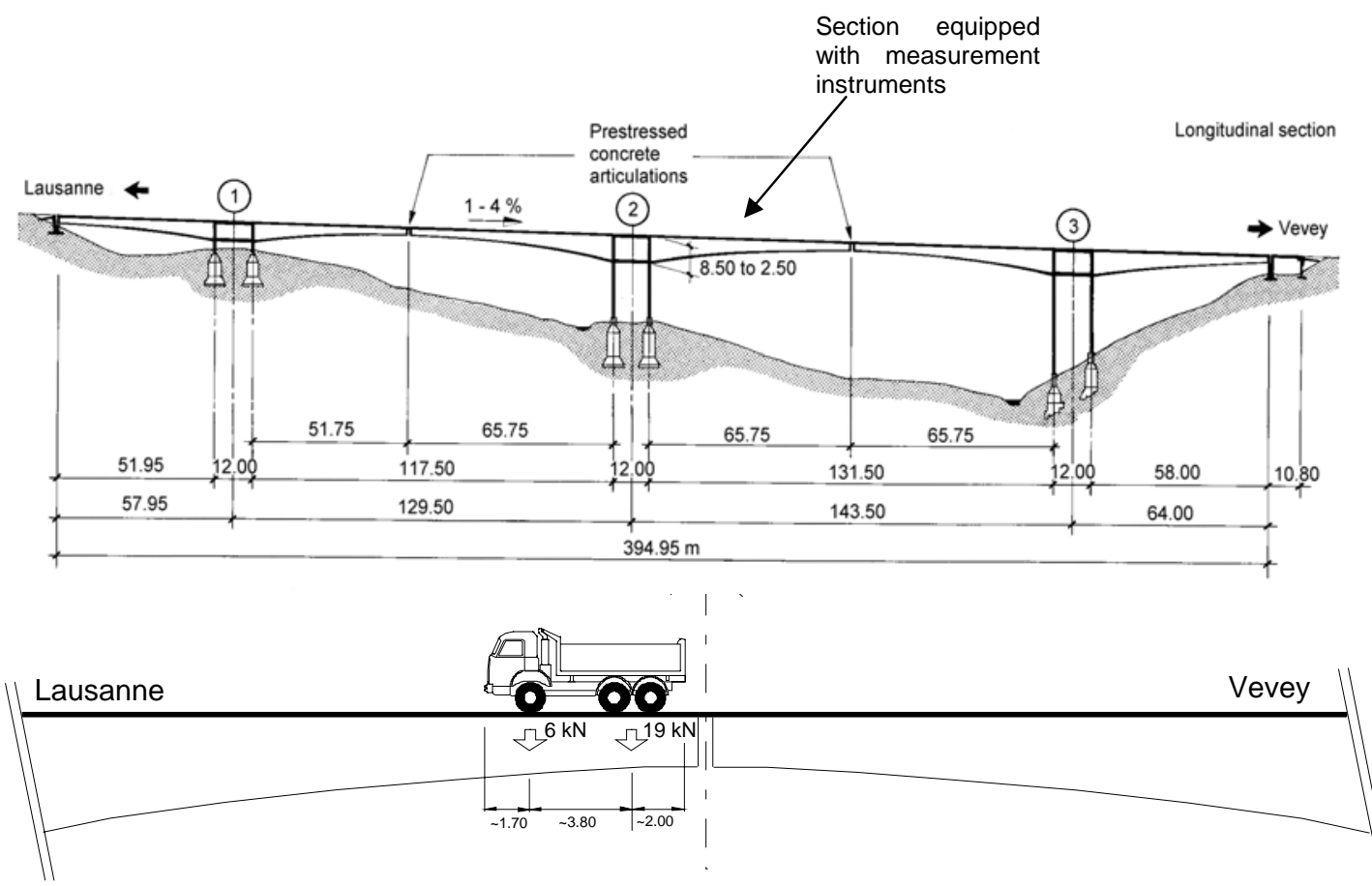

b. Load test on the bridge

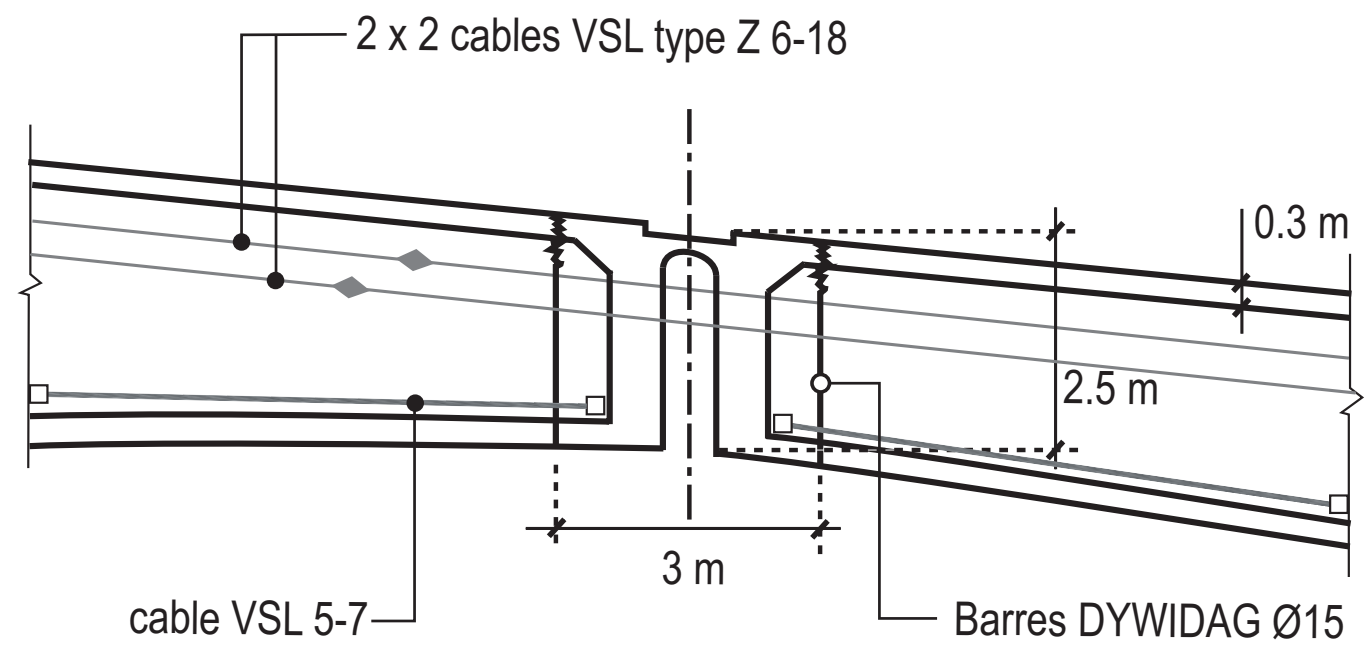

c. Details of the hinge

Figure 7 Lutrive Bridge 


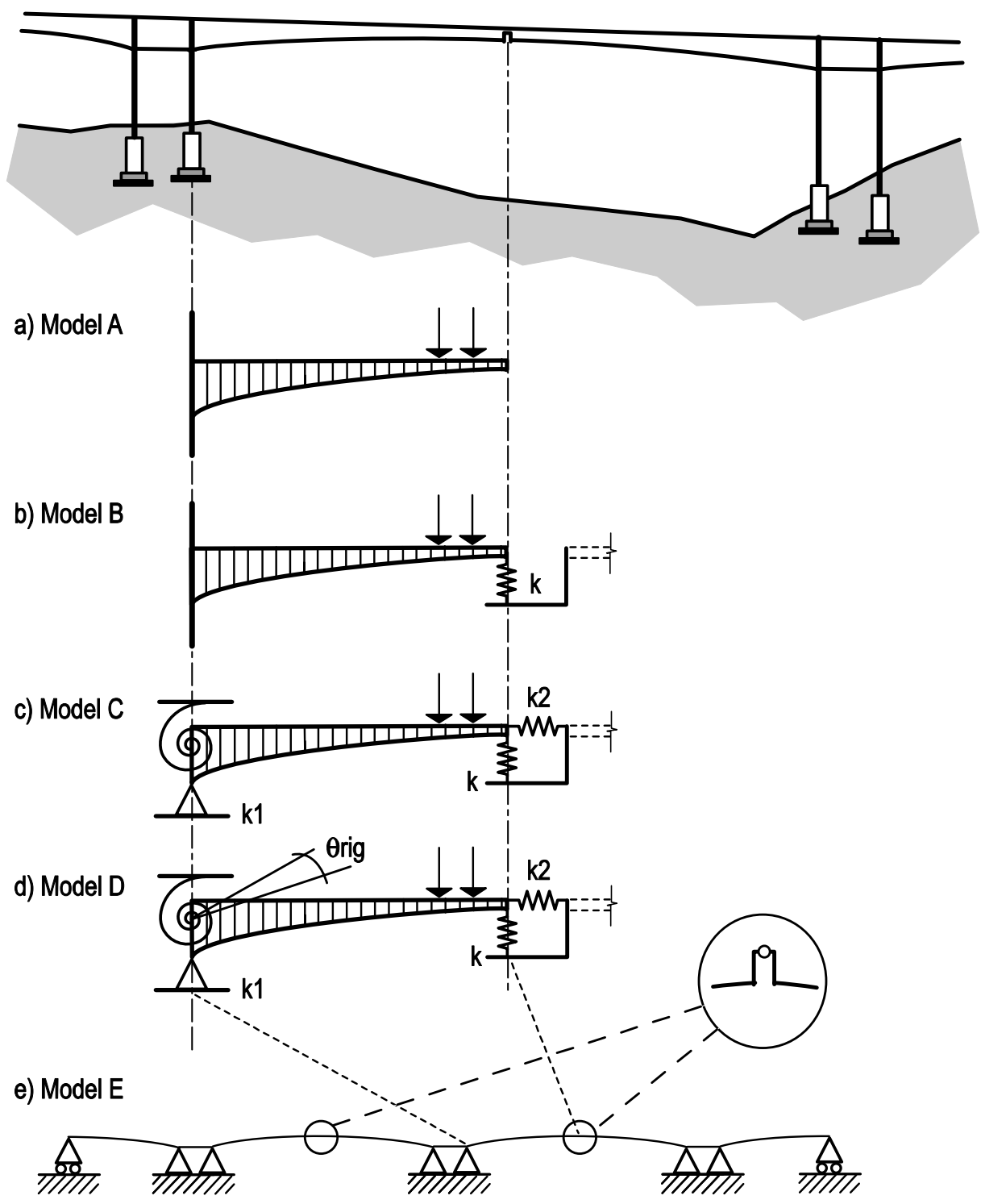

Figure $8 \quad$ Sample set of models examined for the example of the Lutrive bridge 


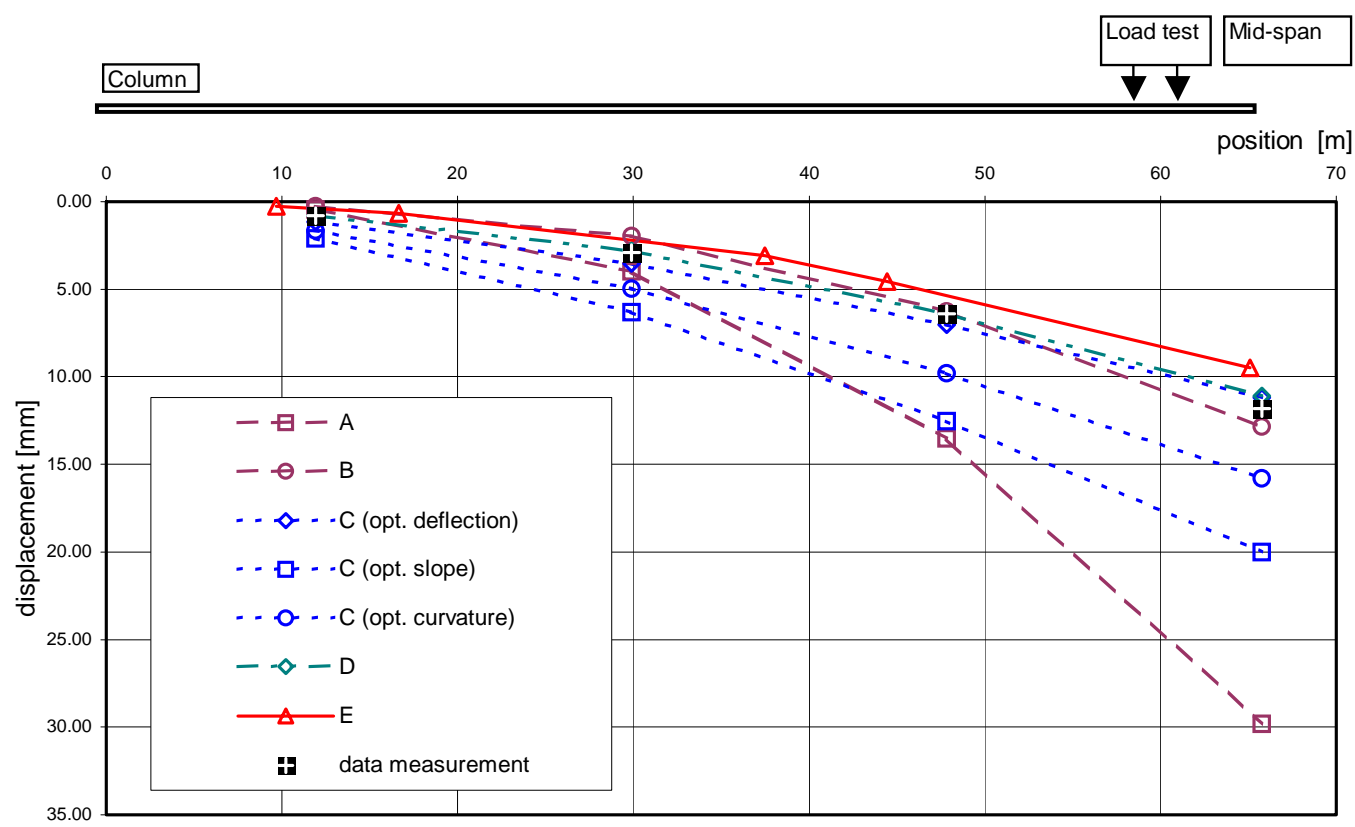

Figure $9 \quad$ Measured and predicted deflections 


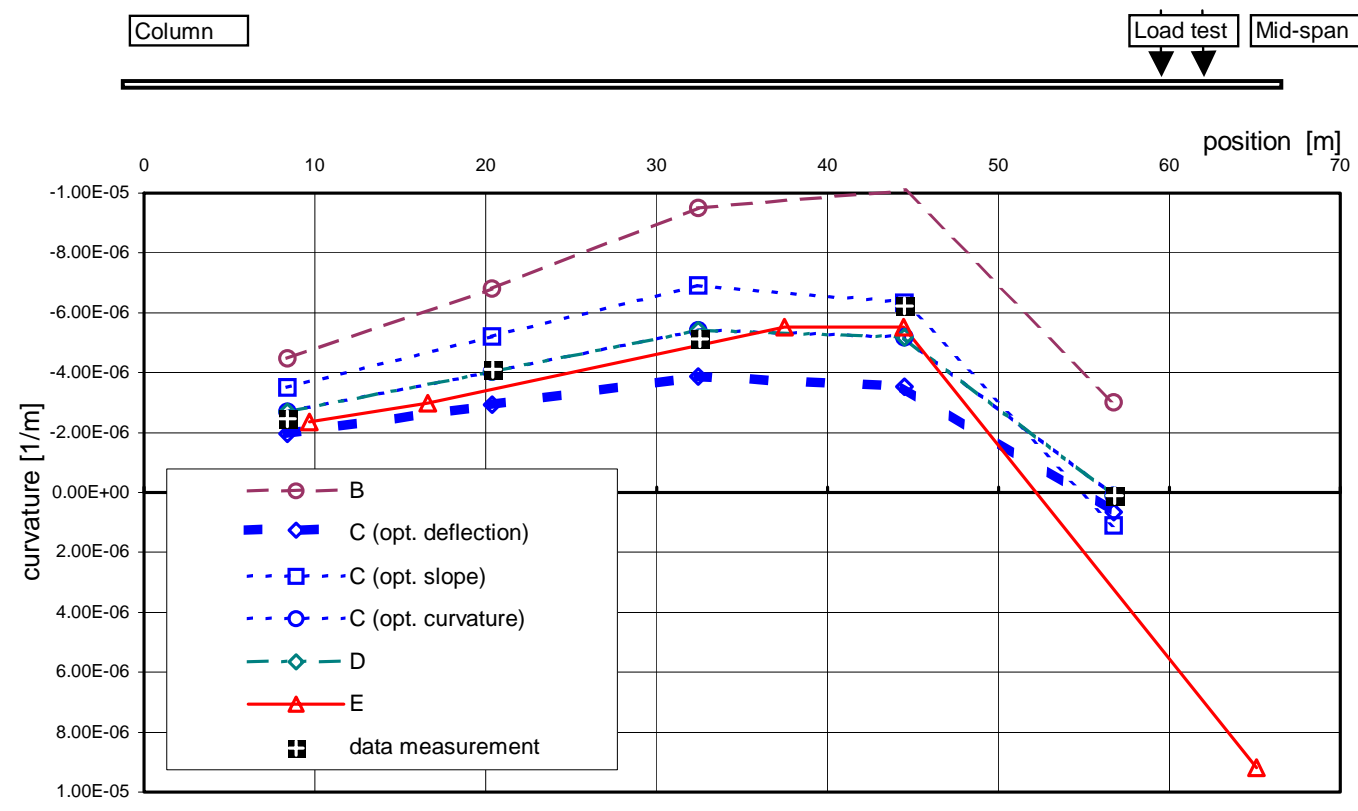

Figure $10 \quad$ Measured and predicted curvatures 


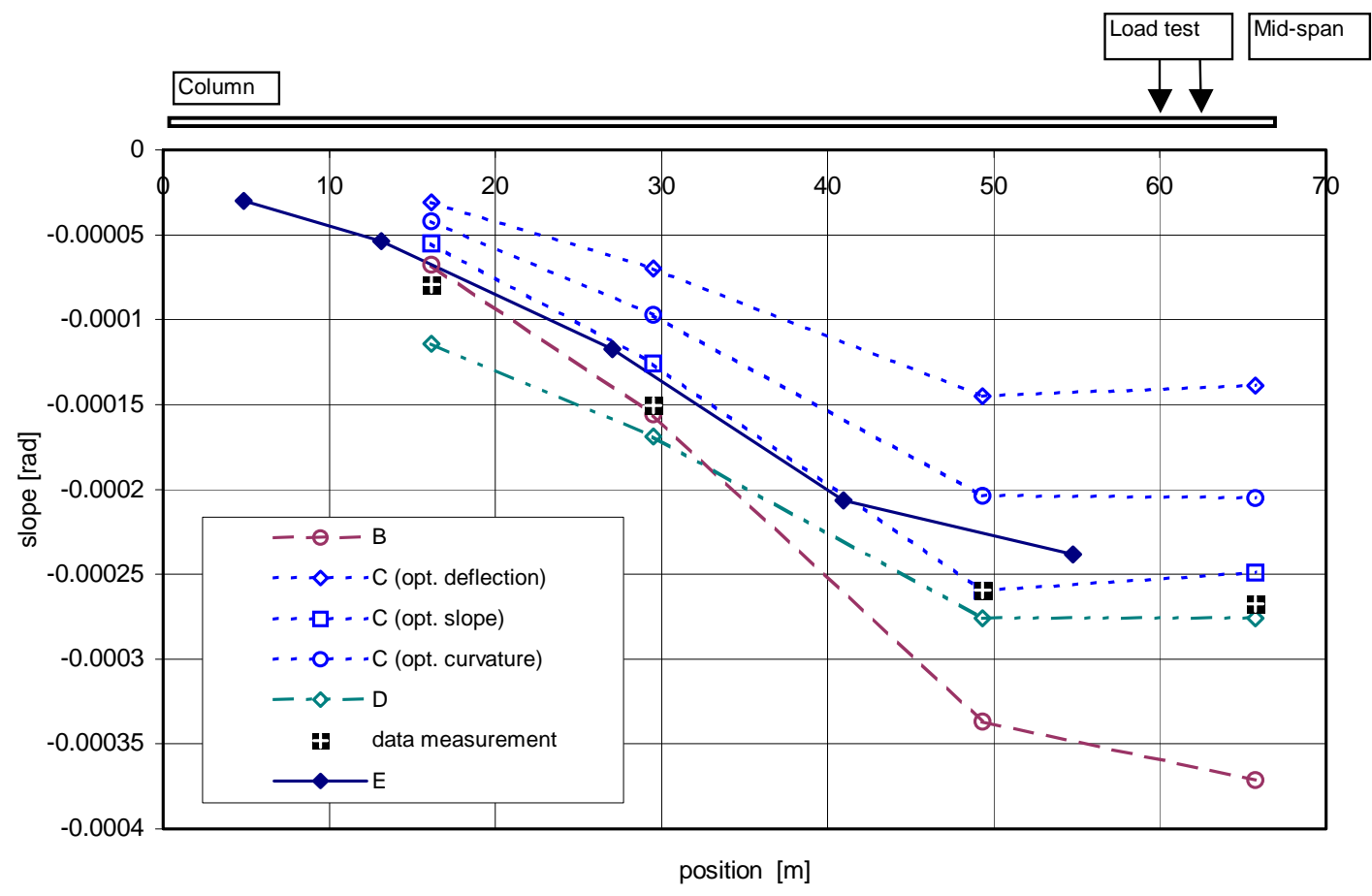

Figure $11 \quad$ Measured and predicted slopes 